PROCEEDINGS OF THE

AMERICAN MATHEMATICAL SOCIETY

Volume 130, Number 9, Pages 2753-2759

S 0002-9939(02)06376-1

Article electronically published on February 12, 2002

\title{
OPEN COLORINGS, THE CONTINUUM AND THE SECOND UNCOUNTABLE CARDINAL
}

\author{
JUSTIN TATCH MOORE
}

(Communicated by Alan Dow)

\begin{abstract}
The purpose of this article is to analyze the cardinality of the continuum using Ramsey theoretic statements about open colorings or "open coloring axioms." In particular it will be shown that the conjunction of two well-known axioms, OCA $\mathbf{A}_{[\mathrm{ARS}]}$ and $\mathbf{O C A}_{[\mathrm{T}]}$, implies that the size of the continuum is $\aleph_{2}$.
\end{abstract}

Our focus in this paper will be the following two open coloring axioms 1 and their influence on the size of the continuum.

$\mathbf{O C A}_{[\mathbf{A R S}]}:$ If $X$ is a separable metric space of size $\aleph_{1}$ and $c:[X]^{2} \rightarrow\{1, \ldots, n\}$ is a continuous map, then there is a decomposition of $X$ into countably many pieces $X_{i}(i \in \mathbb{N})$ such that $c$ is constant on $\left[X_{i}\right]^{2}$ for all $i \in \mathbb{N}$.

$\mathbf{O C A}_{[\mathbf{T}]}:$ If $X$ is a separable metric space and $G \subseteq[X]^{2}$ is open, then either $G$ is countably chromatic (there is a decomposition of $X$ into countably many pieces $X_{i}$ such that $\left[X_{i}\right]^{2} \cap G$ is empty for all $i$ ) or there is an uncountable $H \subseteq X$ such that $[H]^{2} \subseteq G$.

Here $[X]^{2}$ is the set of unordered pairs of distinct elements of $X$. A set $G \subseteq[X]^{2}$ is open if for every $\{x, y\}$ in $G$ there are disjoint neighborhoods $U \ni x$ and $V \ni y$ such that $\left\{\left\{x^{\prime}, y^{\prime}\right\}: x^{\prime} \in U, y^{\prime} \in V\right\}$ is contained in $G$. One defines continuity in a similar way for functions of the form $c:[X]^{2} \rightarrow\{1, \ldots, n\}$.

What we will demonstrate through the course of this note is that the conjunction of these two axioms implies that there are exactly $\aleph_{2}$ real numbers. The proof is divided into four parts, each contributing a section to the paper along with some review and concluding remarks. The first section recalls some of the basic properties of the partial order $\left(\mathbb{N}^{\mathbb{N}},<^{*}\right)$ of eventual dominance. Section 2 gives some of the flavor of the proof from [11] that $\mathbf{O C A}_{[\mathrm{T}]}$ implies that the minimum size of an unbounded subset of $\left(\mathbb{N}^{\mathbb{N}},<^{*}\right)$ is equal to $\aleph_{2}$. Section 3 introduces the alternation map and its properties. This map is then used in Section 4 to code reals inside of certain unbounded subsets of $\left(\mathbb{N}^{\mathbb{N}},<^{*}\right)$ in the presence of $\mathbf{O C A} \mathbf{A}_{[\mathrm{T}]}$. Section 5 shows

Received by the editors March 12, 2001 and, in revised form, April 11, 2001.

2000 Mathematics Subject Classification. Primary 03E65.

Key words and phrases. Open coloring, OCA, continuum problem, oscillation map, alternation map.

The research for this paper was supported by EPSRC grant GR/M71121 during the author's stay at the University of East Anglia; additional support was also received from the Institut Mittag-Leffler during a visit there.

${ }^{1}$ The subscripts $[\mathrm{ARS}]$ and $[\mathrm{T}]$ refer to 1 and [11, where these axioms originally appeared. In the current literature $\mathbf{O C A}$ has come to mean $\mathbf{O C A} \mathbf{A}_{[\mathrm{T}]}$. 
that $\mathbf{O C A}_{[\mathrm{ARS}]}$ prevents too many reals from being coded inside of any subset of $\mathbb{N}^{\mathbb{N}}$ of size $\aleph_{1}$. The final section closes with some questions and comments.

None of the mathematics in this paper should be beyond the understanding of someone able to make sense of the statement of the main theorem. Those familiar with the order $\left(\mathbb{N}^{\mathbb{N}},<^{*}\right)$ should feel free to skip the first section. Those familiar with $\mathbf{O C A} \mathbf{A}_{[\mathrm{T}]}$ may wish to skip directly to Section 3. Readers interested in further reading on $2^{\aleph_{0}}$ and $\aleph_{2}$ are referred to [3], [8], [9], 12], and [13].

\section{The partial order $\left(\mathbb{N}^{\mathbb{N}},<^{*}\right)$}

Our discussion in this paper will frequently focus around the topological space $\mathbb{N}^{\mathbb{N}}$ of all sequences of natural numbers. This is a complete separable metric space when equipped with the product topology. Basic open sets in this context take the form

$$
[u]=\left\{x \in \mathbb{N}^{\mathbb{N}}: x \text { extends } u\right\},
$$

where $u$ is a finite sequence of natural numbers. The complete metric is given by $d(x, y)=2^{-\Delta(x, y)}$, where

$$
\Delta(x, y)=\min \{n: x(n) \neq y(n)\} .
$$

We will also be interested in $\mathbb{N}^{\mathbb{N}}$ as a partially ordered set under the order $<^{*}$ of eventual dominance:

$$
x<^{*} y \text { iff } x(n)<y(n) \text { for all but finitely many } n .
$$

The partial order $\left(\mathbb{N}^{\mathbb{N}},<^{*}\right)$ has a rich structure and frequently shows up in mathematical problems, particularly those close to analysis, set theory, and general topology. The following observations are well known and easily proven.

Fact 1.1. $\left(\mathbb{N}^{\mathbb{N}},<^{*}\right)$ is countably directed (every countable subset has an upper bound).

Fact 1.2. If $X$ is an unbounded and countably directed subset of $\left(\mathbb{N}^{\mathbb{N}},<^{*}\right)$, then whenever $X$ is decomposed into countably many sets $X_{n}(n \in \mathbb{N})$, there is an $n$ such that $X_{n}$ is unbounded and countably directed.

In light of Fact 1.1 and the trivial observation that $\mathbb{N}^{\mathbb{N}}$ is unbounded in $\left(\mathbb{N}^{\mathbb{N}},<^{*}\right)$ it makes sense to define the following cardinal.

Definition 1.3. The unbounding number $\mathfrak{b}$ is the minimum size of an unbounded subset of $\left(\mathbb{N}^{\mathbb{N}},<^{*}\right)$.

This cardinal is therefore somewhere between $\aleph_{1}$ and $2^{\aleph_{0}}$. It turns out to have some rather surprising combinatorial properties (see [11, §1]). The following fact is well known and easily verified.

Fact 1.4. There is an unbounded chain in $\left(\mathbb{N}^{\mathbb{N}},<^{*}\right)$ of order type $\mathfrak{b}$. In particular, there is an unbounded and countably directed subset of $\left(\mathbb{N}^{\mathbb{N}},<^{*}\right)$ of size $\mathfrak{b}$.

The next observation is behind much of the combinatorics associated with unboundedness in $\left(\mathbb{N}^{\mathbb{N}},<^{*}\right)$.

Fact 1.5. Suppose $X \subseteq \mathbb{N}^{\mathbb{N}}$ is nonempty and has the property that every neighborhood of $X$ is unbounded. For every basic open set $[u]$, if $[u] \cap X$ is nonempty, then there is a $v$ extending $u$ such that $\left[v^{\wedge} i\right] \cap X$ is nonempty for infinitely many $i$. 
Notice that since the union of countably many bounded sets is bounded (by Fact 1.1), if $X$ is an unbounded subset of $\left(\mathbb{N}^{\mathbb{N}},<^{*}\right)$ then it contains a nonempty set $X^{\prime}$ such that every neighborhood of $X^{\prime}$ is unbounded.

\section{2. $\aleph_{2}$ AND THE UNBOUNDING NUMBER}

This section will review the following result and give a sketch of its proof.

Theorem 2.1 ([11]). OCA $\mathbf{A T}_{[\mathrm{T}]}$ implies that $\mathfrak{b}$ is $\aleph_{2}$.

This result breaks into two parts, one showing $\mathfrak{b}$ is at least $\aleph_{2}$ and the other showing that it is at most $\aleph_{2}$. Proving that $\mathfrak{b}$ is at least $\aleph_{2}$ under OCA $\mathbf{A}_{[\mathrm{T}]}$ was done originally via the oscillation map (see [1]). It was this proof that led me to the discovery of the alternation map (which is essentially a continuous version of the oscillation map) and to the coding technique which will be presented in the later sections. An interested reader is encouraged to deduce this portion of Theorem 2.1 from the properties of the alternation map given below.

The half of Theorem [2.1] which deals with the inequality $\mathfrak{b} \leq \aleph_{2}$ makes crucial use of the following well-studied notion.

Definition 2.2. If $\kappa$ and $\lambda$ are cardinals, then a $\left(\kappa, \lambda^{*}\right)$ gap in $\left(\mathbb{N}^{\mathbb{N}},<^{*}\right)$ is a pair of sequences $a_{\xi}(\xi<\kappa)$ and $b_{\eta}(\eta<\lambda)$ in $\mathbb{N}^{\mathbb{N}}$ such that

$$
a_{\xi}<^{*} a_{\xi^{\prime}}<^{*} b_{\eta^{\prime}}<^{*} b_{\eta}
$$

for all $\xi<\xi^{\prime}<\kappa$ and $\eta<\eta^{\prime}<\lambda$, but for which there is no single $c$ in $\mathbb{N}^{\mathbb{N}}$ such that $a_{\xi}<^{*} c<^{*} b_{\eta}$ for all $\xi<\kappa$ and $\eta<\lambda$.

Long ago Hausdorff made the following important connection between gaps and unbounded chains in $\left(\mathbb{N}^{\mathbb{N}},<^{*}\right)$.

Theorem 2.3 ([4]). If $\kappa$ is a regular cardinal, then there is an unbounded chain in $\left(\mathbb{N}^{\mathbb{N}},<^{*}\right)$ of order type $\kappa$ iff there is a $\left(\kappa, \omega^{*}\right)$ gap in $\left(\mathbb{N}^{\mathbb{N}},<^{*}\right)$.

Hausdorff also discovered another type of gap — the $\left(\omega_{1}, \omega_{1}^{*}\right)$ gap. Rather remarkably, this is the best that one can do without using extra assumptions.

Theorem 2.4 ([1] $)$. Under $\mathbf{O C A} \mathbf{A}_{[\mathrm{T}]}$ all gaps in $\left(\mathbb{N}^{\mathbb{N}},<^{*}\right)$ are of the form $\left(\omega_{1}, \omega_{1}^{*}\right)$, $\left(\kappa, \omega^{*}\right)$, or $\left(\omega^{*}, \kappa\right)$ for some cardinal $\kappa$.

Also quite striking is the following fact, which now completes the proof of Theorem 2.1. An interested reader is encouraged to supply its proof via Theorem [2.3.

Lemma 2.5 (11]). If every subset of $\mathbb{N}^{\mathbb{N}}$ of size at most $\aleph_{2}$ is bounded, then there is an $\left(\omega_{2}, \kappa^{*}\right)$ gap in $\left(\mathbb{N}^{\mathbb{N}},<^{*}\right)$ for some regular uncountable cardinal $\kappa$.

\section{The Alternation MAP}

For technical reasons we will now restrict our discussion to the suborder $\left(\mathbb{N}^{\uparrow \mathbb{N}},<^{*}\right)$ of strictly increasing sequences of natural numbers. Since $\mathbb{N}^{\uparrow} \mathbb{N}$ is both cofinal in and order isomorphic to $\left(\mathbb{N}^{\mathbb{N}},<^{*}\right)$, this is really not a loss of generality. The following notion will be central to our discussion in this section.

Definition 3.1. Suppose that $S$ is a subset of $\mathbb{N}$. Two sequences $x$ and $y$ in $\mathbb{N}^{\uparrow \mathbb{N}}$ are said to alternate on $S$ if $x(n) \neq y(n)$ for all $n$ in $S$ and, for every pair of consecutive elements $m$ and $n$ of $S, x(m)<y(m)$ iff $x(n)>y(n)$. 
Notice that this notion also makes sense if $x$ and $y$ are only partial functions whose domains include $S$.

Now suppose that $x$ and $y$ are two distinct elements of $\mathbb{N}^{\uparrow \mathbb{N}}$ and that $x$ is lexicographically less than $y$. Define a set $S(x, y) \subseteq \mathbb{N}$ by recursion. The first element of $S(x, y)$ is $\Delta(x, y)$. Given the $2 n^{\text {th }}$ element $k$ of $S(x, y)$, define the next two elements to be $y(k)$ and $x(y(k))$. So the elements of $S(x, y)$ are

$$
\Delta(x, y), y(\Delta(x, y)), x(y(\Delta(x, y))), y(x(y(\Delta(x, y)))), \ldots .
$$

If $x$ and $y$ are only partial functions, repeat this procedure so long as the elements generated remain in the domains of both $x$ and $y$. Since $x$ and $y$ are strictly increasing, the elements of $S$ being defined are getting successively larger and larger. If $i$ is an integer and $S(x, y)$ has an $i^{\text {th }}$ element (starting with $i=0$ ), then we will occasionally find it convenient to denote it as $S(x, y)(i)$.

Notice that the map $S:\left[\mathbb{N}^{\uparrow \mathbb{N}}\right]^{2} \rightarrow \mathcal{P}(\mathbb{N})$ is continuous, since to determine a finite amount of information about $S(x, y)$ one needs only a finite amount of information about $x$ and $y$. Given $S(x, y)$, alt $(x, y)$ is defined to be the cardinality of the largest initial segment of $S(x, y)$ on which $x$ and $y$ alternate, minus one. Thus alt : $\left[\mathbb{N}^{\uparrow \mathbb{N}}\right]^{2} \rightarrow \mathbb{N} \cup\{\infty\}$ is also a continuous map, where $\mathbb{N} \cup\{\infty\}$ is the one-point compactification of $\mathbb{N}$. Notice that the alternation map is finite on all pairs which are comparable in $\left(\mathbb{N}^{\uparrow \mathbb{N}},<^{*}\right)$.

The following theorem shows that the alternation map exhibits a behavior similar to that of the better-known oscillation map of Todorčević (see, e.g., 11). It has essentially appeared in [5] and chapter 4 of [6]. A proof has been included for completeness.

Theorem 3.2. If $X$ is unbounded and countably directed in $\left(\mathbb{N}^{\uparrow \mathbb{N}},<^{*}\right)$, then there is an $n$ such that for every $k$ there is a pair $x, y$ in $X$ with $\Delta(x, y)=n$ and $\operatorname{alt}(x, y)=k$.

Proof. Let $X$ be given and choose a countable dense subset $D$ of $X$. Since $\left(X,<^{*}\right)$ is countably directed, there is an $a$ in $X$ which is an upper bound for $D$. Now consider the set of all $\left\{y \in X: a<^{*} y\right\}$. Since $X$ is directed and unbounded, this set is also unbounded. It is therefore possible by Fact 1.2 to find an $n$ and a subcollection $Y$ of this set such that every neighborhood of $Y$ is unbounded and $a(i)<y(i)$ for every $y$ in $Y$ and $i \geq m$.

Now choose a sequence $u_{k}(k \in \mathbb{N})$ such that for all $k$

1. $u_{k}$ is a finite sequence of natural numbers such that $\left[u_{k}{ }^{\wedge} i\right] \cap Y$ is nonempty for infinitely many $i$,

2. $u_{k}$ is an initial segment of $u_{k+2}$,

3. $\Delta\left(u_{k}, u_{k+1}\right)=\left|u_{0}\right|>m$,

4. $\left|u_{k}\right| \leq n_{k}<\left|u_{k+1}\right|$, and

5. $u_{k+2}\left(n_{k}\right)<u_{k+1}\left(n_{k}\right)$, where $n_{k}=S\left(u_{k+1}, u_{k+2}\right)(k)$.

The recursion is easily carried out using Fact [1.5] Notice that $u_{2 k+1}<_{\operatorname{lex}} u_{2 k+2}$ follows from 2, 3, and [5. Also, by 2 and 3, $S\left(u_{k+1}, u_{k+2}\right)(i)$ is a fixed integer $n_{i}$ which does not depend on $k$ provided that $i \leq k$. By 5, $u_{k}$ and $u_{k+1}$ alternate on $S\left(u_{k}, u_{k+1}\right)=\left\{n_{0}, \ldots, n_{k-1}\right\}\left(S\left(u_{0}, u_{1}\right)\right.$ is empty $)$.

Now let $d_{k}$ be in $\left[u_{k+1}\right] \cap D$ and fix an $m_{k}$ such that $d_{k}(i)<a(i)$ for all $i>m_{k}$. Choose $y_{k}$ in $\left[u_{k}\right] \cap Y$ such that $y_{k}\left(\left|u_{k}\right|\right)>a\left(m_{k}\right)$. Note that $y_{k}$ dominates $d_{k}$ on the interval $\left[\left|u_{k}\right|, m_{k}\right]$ by monotonicity, and $y_{k}$ dominates $d_{k}$ on $\left[m_{k}, \infty\right)$ since $m_{k}>m$. It follows that $\Delta\left(d_{k}, y_{k}\right)=n_{0}$ and that $\left\{n_{0}, \ldots, n_{k}\right\}$ are the first $k+1$ elements of 
$S\left(d_{k}, y_{k}\right)$. Note that $y_{k}\left(n_{k}\right)>d_{k}\left(n_{k}\right)$ and that by 4 the next element of $S\left(d_{k}, y_{k}\right)$ is at least $\left|u_{k}\right|$, and hence $y_{k}$ dominates $d_{k}$ at this place as well. By our observations above, $\left\{n_{0}, \ldots, n_{k}\right\}$ is exactly the largest initial segment of $S\left(d_{k}, y_{k}\right)$ on which $d_{k}$ and $y_{k}$ alternate, making alt $\left(d_{k}, y_{k}\right)=k$.

\section{Continuously Coding a Real number}

In this section we will see how to code countable binary sequences into subsets of $\mathbb{N}^{\mathbb{N}}$. Since the real numbers have the same size as $\{0,1\}^{\mathbb{N}}$, this can be considered as a means for coding reals. To facilitate the coding that we are about to define, it is useful if we change the map alt to a map $t$ which takes values which are finite binary sequences rather than natural numbers. To this end let $\varphi(k, n)$ be the unique binary sequence $t$ of length $n$ such that

$$
k \equiv \sum_{i=0}^{n-1} t(i) 2^{i} \quad \bmod 2^{n} .
$$

It is easily seen that $\varphi(\cdot, n)$ maps onto the binary sequences of length $n$.

If $x, y$ are in $\mathbb{N}^{\uparrow \mathbb{N}}$ and $\operatorname{alt}(x, y)$ is finite, define

$$
t(x, y)=\varphi(\operatorname{alt}(x, y), \Delta(x, y)) .
$$

Notice that, since it is a composition of continuous functions, $t$ is continuous on its domain. Its domain is the preimage of $\mathbb{N}$ under the alternation map, and is therefore an open set. Similarly, $t$ is defined on all pairs $x$ and $y$ which are comparable in $\left(\mathbb{N}^{\uparrow \mathbb{N}},<^{*}\right)$. Theorem 3.2 immediately gives the following result.

Theorem 4.1. If $X$ is unbounded and countably directed in $\left(\mathbb{N}^{\uparrow \mathbb{N}},<^{*}\right)$ and $r$ is in $\{0,1\}^{\mathbb{N}}$, then there is a pair $x, y$ in $X$ such that $t(x, y)$ is defined and is an initial segment of $r$.

Now we are ready to define what it means for a set $H \subseteq \mathbb{N}^{\uparrow \mathbb{N}}$ to code an element $r$ of $\{0,1\}^{\mathbb{N}}$.

Definition 4.2. If $H \subseteq \mathbb{N}^{\uparrow \mathbb{N}}$ is uncountable, $t$ is defined on $[H]^{2}$, and the image of $[H]^{2}$ under $t$ contains no two incompatible elements, then $H$ is said to be a code. If $r$ is the unique element of $\{0,1\}^{\mathbb{N}}$ such that $t(x, y)$ is an initial segment of $r$ for all $x, y$ in $H$, then we will say that $r$ is coded by $H$.

Remark 4.3. Notice that the map $\Delta$ is unbounded on the pairs of any uncountable set $X$. This implies that no set can be a code for two distinct $r$ and $r^{\prime}$ in $\{0,1\}^{\mathbb{N}}$.

Theorem $4.4\left(\mathbf{O C A}_{[\mathrm{T}]}\right)$. If $X \subseteq \mathbb{N}^{\uparrow \mathbb{N}}$ is unbounded and countably directed, then for every $r$ in $\{0,1\}^{\mathbb{N}}$ there is an $H \subseteq X$ which codes it.

Proof. Define $G_{r} \subseteq[X]^{2}$ by putting $\{x, y\}$ in $G_{r}$ iff $t(x, y)$ is defined and is an initial segment of $r$. Since $t$ is continuous and has an open domain, $G_{r}$ is open. By Fact 1.2 and Theorem $4.1 G_{r}$ isn't countably chromatic. Applying $\mathbf{O C A} \mathbf{A}_{[\mathrm{T}]}$, there is an uncountable $H \subseteq X$ such that $[H]^{2} \subseteq G_{r}$, which, by definition, is a code for $r$. 


\section{SEAling CODES}

In this section we finish the proof of the main result by showing that under $\mathbf{O C A}_{[\mathrm{ARS}]}$ any chain in $\left(\mathbb{N}^{\uparrow \mathbb{N}},<^{*}\right)$ of size $\aleph_{2}$ can contain codes for at most $\aleph_{2}$ reals.

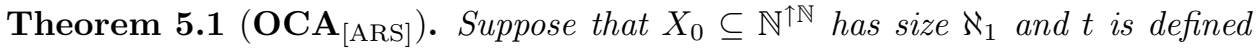
on $\left[X_{0}\right]^{2}$. There is a pairwise disjoint family $A_{r}\left(r \in\{0,1\}^{\mathbb{N}}\right)$ of (possibly empty) subsets of $X_{0}$ such that if $H \subseteq X_{0}$ is a code for $r$, then $H \backslash A_{r}$ is at most countable. In particular, at most $\aleph_{1}$ many different elements of $\{0,1\}^{\mathbb{N}}$ have codes contained in $X_{0}$.

Proof. Define $t_{n}:\left[X_{0}\right]^{2} \rightarrow\{0,1\}^{\leq n}$ for each $n$ by setting $t_{n}(x, y)$ to be the restriction of $t(x, y)$ to its first $n$ entries (set $t_{n}(x, y)=t(x, y)$ if $\left.\Delta(x, y) \leq n\right)$. Note that $t_{n}$ is also continuous for each $n$ and has finite range. Construct a family $\left\{A_{s}\right\}$ indexed by the finite binary sequences with the following properties:

1. For each $n$ the collection $\left\{A_{s}: s \in\{0,1\}^{n}\right\}$ is a partition of $X_{0}$ into disjoint sets.

2. Each $A_{s}$ can be decomposed into countably many sets $A_{s, i}$ such that $t_{|s|}$ is constantly $s$ on $\left[A_{s, i}\right]^{2}$ for all $i$.

Let $n$ be fixed and apply $\mathbf{O C A}_{[\mathrm{ARS}]}$ to $t_{n}:\left[X_{0}\right]^{2} \rightarrow\{0,1\}^{\leq n}$ to get countably many sets $Y_{i}$ such that $t_{n}$ is constant on $\left[Y_{i}\right]^{2}$ for all $i$. Notice we may assume that, for each $i$, if $x \neq y$ are in $Y_{i}$, then $\Delta(x, y)>n$. It follows that for every $i$ there is an $s$ in $\{0,1\}^{n}$ such that $t$ is constantly $s$ on $\left[Y_{i}\right]^{2}$ (possibly for trivial reasons). The sets $A_{s}\left(s \in\{0,1\}^{n}\right)$ are now constructed so that they are pairwise disjoint and each $A_{s}$ is contained in union of all $Y_{i}$ such that $t_{n}$ is constantly $s$ on $\left[Y_{i}\right]^{2}$. It should be clear that these sets satisfy properties 1 and 2 above.

If $r$ is in $\{0,1\}^{\mathbb{N}}$, set $A_{r}=\bigcap_{n=0}^{\infty} A_{r \uparrow n}$. By property $1, A_{r}$ and $A_{r^{\prime}}$ are disjoint for distinct $r$ and $r^{\prime}$ in $\{0,1\}^{\mathbb{N}}$. Now observe that, by property 2 , if $s \in\{0,1\}^{n}$ is different than $r \uparrow n$, then $H \cap A_{s}$ is countable since $t_{n}^{\prime \prime}[H]^{2} \subseteq\{\langle\rangle, \ldots, r \uparrow n\}$. Applying property 1 again, we see that $H \backslash A_{r\lceil n}$ is countable for all $n$, and hence $H \backslash A_{r}$ is countable.

\section{Closing Remarks}

We will now close this paper by mentioning the following two open problems.

Question 6.1. Does OCA $\mathbf{O A R S}_{[\mathrm{Amply}} 2^{\aleph_{0}}$ is $\aleph_{2}$ ?

Question 6.2. Does $\mathrm{OCA}_{[\mathrm{T}]}$ imply $2^{\aleph_{0}}$ is $\aleph_{2}$ ?

Both of these questions are interesting for different reasons and, in spite of their similar appearance, seem to be quite different in nature. Question 6.1 is a special case of the better-known open problem of whether the Bounded Proper Forcing Axiom implies $2^{\aleph_{0}}=\aleph_{2}$ (see [10] for more information on bounded forcing axioms). Question 6.2 has been pursued since $\mathbf{O C A}_{[\mathrm{T}]}$ was introduced. Interest in it stems both from the wide variety of applications which this axiom has seen (see, e.g., [2], [11, §8]) and from the fact that it is consistent with an effective form of the Continuum Hypothesis in the absence of the Axiom of Choice (see [7]). I. Farah has shown in an unpublished note that it is consistent that $\mathbf{O C \mathbf { A } _ { [ \mathrm { T } ] }}$ holds for graphs of size $\aleph_{1}$ and $2^{\aleph_{0}}>\aleph_{2}$, but it is known that this restricted form of the axiom is much weaker than $\mathbf{O C A}_{[\mathrm{T}]}$ (see [10]). Both questions are also interesting if they are supplemented with $\mathbf{M} \mathbf{A}_{\aleph_{1}}$, an axiom often used in conjunction with these axioms. 
Any complete resolution to these questions would be of great interest. If either question has a positive answer (particularly in the case of Question 6.1), this would seem to require a new method for proving that $2^{\aleph_{0}}$ is $\aleph_{2}$. On the other hand, if both questions have a negative answer, this would give two Ramsey theoretic statements which are individually consistent with $2^{\aleph_{0}}>\aleph_{2}$ but which jointly imply $2^{\aleph_{0}}=\aleph_{2}$. Situations of this kind (and lack thereof) have recently received a great deal of attention - see [13].

\section{ACKNOWLEDGEMENTS}

The author thanks Stevo Todorčević for providing Theorem 2.1 as an exercise back in 1997. He also offered some useful suggestions on the presentation of the material in Section 2 .

\section{REFERENCES}

[1] U. Abraham, M. Rubin, S. Shelah. On the Consistency of Some Partition Theorems for Continuous Colorings, and the Structure of $\aleph_{1}$-dense Real Order Types. Ann. Pure Appl. Logic 292 (1985) 123-206. MR 87d:03132

[2] I. Farah. Analytic Quotients. Memoirs of the AMS 148702 (2000). MR 2001c:03076

[3] M. Foreman, M. Magidor, S. Shelah. Martin's Maximum, Saturated Ideals, and Nonregular Ultrafilters. I. Ann. of Math. 1271 (1988) 1-47. MR 89f:03043

[4] F. Hausdorff. Die Graduierung nach dem Endverlauf. Abhandlun. König. Sächs. Gesellsch. Wissenschaften, Math.-Phys. Kl. 31 (1909) 296-334.

[5] J. T. Moore. Continuous Colorings Associated With Certain Characteristics of the Continuum. Discrete Math. 214 (2000) 263-273. MR 2001f:03094

[6] J. T. Moore. Topics in Ramsey Theory on Sets of Real Numbers. Ph.D. Thesis. University of Toronto (2000).

[7] C. Di Prisco, S. Todorčević. Perfect Set Properties in $\mathbf{L}(\mathbb{R})[U]$. Adv. Math. 1392 (1998) 240-259. MR 99m:03102

[8] S. Todorčević. Comparing the Continuum With the First Two Uncountable Cardinals, in Logic and Scientific Methods (M. L. Dalla, et al, eds.). Kluwer Acad. Publ. (1997) 145-155. CMP 2001:05

[9] S. Todorčević. Conjectures of Chang and Rado and Cardinal Arithmetic, in Finite and Infinite Sets in Combinatorics (N. W. Sauer et al., eds), Kluwer Acad. Publ. (1993) 385-398. MR 95h:03113

[10] S. Todorčević. Localized reflection and fragments of PFA. DIMACS Ser. Discrete Math. Theoret. Comput. Sci. (to appear).

[11] S. Todorčević. Partition Problems In Topology. American Math. Soc., Providence (1989). MR 90d:04001

[12] B. Veličković. Forcing Axioms and Stationary Sets. Adv. Math. 94 (1992) 256-284. MR 93k:03045

[13] W. H. Woodin. The Axiom of Determinacy, Forcing Axioms, and the Nonstationary Ideal. de Gruyter Series in Logic and its Applications (1999). MR 2001e:03001

Department of Mathematics, Boise State University, Boise, Idaho 83725

E-mail address: justin@math.boisestate.edu 